Pacific

Journal of

Mathematics

\title{
ON THE TORSION ANOMALOUS CONJECTURE IN CM ABELIAN VARIETIES
}

\author{
SARa Checcoli AND Evelina Viada
}




\title{
ON THE TORSION ANOMALOUS CONJECTURE IN CM ABELIAN VARIETIES
}

\author{
SARA CHECCOLI AND Evelina ViadA
}

\begin{abstract}
The torsion anomalous conjecture (TAC) states that a subvariety $V$ of an abelian variety $\boldsymbol{A}$ has only finitely many maximal torsion anomalous subvarieties. In this work we prove, with an effective method, some cases of the TAC when the ambient variety $\boldsymbol{A}$ has $\mathrm{CM}$, generalising our previous results in products of $\mathrm{CM}$ elliptic curves. When $V$ is a curve, we give new results and we deduce some implications on the effective Mordell-Lang conjecture.
\end{abstract}

\section{Introduction}

Let $A$ be an abelian variety embedded in the projective space and let $V$ be a proper subvariety of $A$. Assume that both $A$ and $V$ are defined over the algebraic numbers.

Definition 1.1. The variety $V$ is a translate (resp. a torsion variety) if it is a finite union of translates of proper algebraic subgroups by points (resp. by torsion points).

$V$ is transverse (resp. weak-transverse) in $A$ if $V$ is irreducible and $V$ is not contained in any translate (resp. in any torsion subvariety) of $A$.

It is a classical problem in diophantine geometry to investigate the relationship between the above geometrical definitions and the arithmetical properties of the variety $V$. In this direction, there are several celebrated theorems, such as the Manin-Mumford, Mordell-Lang and Bogomolov conjectures.

Recently E. Bombieri, D. Masser and U. Zannier [Bombieri et al. 2007] suggested a new approach to this kind of investigation, introducing in particular the notion of torsion anomalous intersections.

Definition 1.2. An irreducible subvariety $Y$ of $V$ is $V$-torsion anomalous if:

- $Y$ is an irreducible component of $V \cap(B+\zeta)$, with $B+\zeta$ an irreducible torsion variety.

- The dimension of $Y$ is larger than expected; i.e.,

$$
\operatorname{codim} Y<\operatorname{codim} V+\operatorname{codim} B \text {. }
$$

Keywords: diophantine approximation, heights, abelian varieties, intersections with torsion varieties. 
The variety $B+\zeta$ is minimal for $Y$ if, in addition, it has minimal dimension. The relative codimension of $Y$ is the codimension of $Y$ in such a minimal $B+\zeta$.

We say that $Y$ is maximal if it is not contained in any $V$-torsion anomalous variety of strictly larger dimension.

In [Bombieri et al. 2007], the authors formulate several conjectures. We state here one natural variant.

Conjecture 1.3 (TAC, torsion anomalous conjecture). For any algebraic subvariety $V$ of a (semi)abelian variety, there are only finitely many maximal $V$-torsion anomalous varieties.

The TAC is well known to have several important consequences. It implies, for instance, the Manin-Mumford and the Mordell-Lang conjectures; it is also related to model theory by the work of B. Zilber and to algebraic dynamics by the recent work of J. H. Silverman and P. Morton. In addition, R. Pink generalised it to mixed Shimura varieties.

Only a few cases of the TAC are known: Viada [2008] proved it for curves in a product of elliptic curves, Maurin [2008] for curves in a torus, Bombieri et al. [2007] for varieties of codimension 2 in a torus. Habegger [2008] gave related results under some stronger assumptions on $V$.

In [Checcoli et al. 2014], we prove an effective TAC for maximal $V$-torsion anomalous varieties of relative codimension 1 in a product of $\mathrm{CM}$ elliptic curves. Our bounds are explicit and uniform in their dependence on $V$. As an immediate corollary, we prove the TAC for varieties of codimension 2, obtaining an elliptic analogue of the toric result in [Bombieri et al. 2007]. In the present work, we generalise our results to CM abelian varieties. In [Checcoli et al. 2014], we also point out interesting relations between this kind of theorem and other relevant conjectures, such as the Zilber-Pink conjecture and the above-mentioned ones.

Let $A \subseteq \mathbb{P}^{m}$ be an abelian variety with CM defined over a number field $k$ and let $k_{\text {tor }}$ be the field of definition of the torsion points of $A$. Let $A$ be isogenous to a product of simple abelian varieties of dimension at most $g$. For a point $x \in A$, we denote by $\hat{h}(x)$ its canonical Néron-Tate height. For a subvariety $V \subseteq A$, we denote by $h(V)$ its normalised height and by $k_{\text {tor }}(V)$ its field of definition over $k_{\text {tor }}$ (see Section 2). By « we denote an inequality up to a multiplicative constant depending on $A$. Our main result is the following:

Theorem 1.4. Let $V \subseteq A$ be a weak-transverse subvariety of codimension $>g$. Then there are only finitely many maximal $V$-torsion anomalous subvarieties $Y$ of relative codimension 1 .

Effective version: More precisely, if $B+\zeta$ is minimal for $Y$, then for any positive real $\eta$, there exist constants depending only on $A$ and $\eta$ such that: 
(1) If $Y$ is not a translate, then

$$
\begin{aligned}
\operatorname{deg} B & \ll_{\eta}(h(V)+\operatorname{deg} V)^{\operatorname{codim} B+\eta}, \\
h(Y) & \ll_{\eta}(h(V)+\operatorname{deg} V)^{\operatorname{codim} B+\eta}, \\
\operatorname{deg} Y & \ll_{\eta} \operatorname{deg} V(h(V)+\operatorname{deg} V)^{\operatorname{codim} B-1+\eta} .
\end{aligned}
$$

(2) If $Y$ is a point, then

$$
\begin{aligned}
\operatorname{deg} B & \ll_{\eta}\left((h(V)+\operatorname{deg} V)\left[k_{\mathrm{tor}}(V): k_{\mathrm{tor}}\right]\right)^{\operatorname{codim} B+\eta}, \\
\hat{h}(Y) & \ll_{\eta}(h(V)+\operatorname{deg} V)^{\operatorname{codim} B+\eta}\left[k_{\mathrm{tor}}(V): k_{\mathrm{tor}}\right]^{\operatorname{codim} B-1+\eta}, \\
{\left[k_{\mathrm{tor}}(Y): k_{\mathrm{tor}}\right] } & \ll_{\eta} \operatorname{deg} V\left[k_{\mathrm{tor}}(V): k_{\mathrm{tor}}\right]^{\operatorname{codim} B+\eta}(h(V)+\operatorname{deg} V)^{\operatorname{codim} B-1+\eta} .
\end{aligned}
$$

(3) If $Y$ is a translate of positive dimension, then

$$
\begin{gathered}
\operatorname{deg} B \ll_{\eta}\left((h(V)+\operatorname{deg} V)\left[k_{\mathrm{tor}}(V): k_{\mathrm{tor}}\right]\right)^{\operatorname{codim} B+\eta}, \\
h(Y) \ll_{\eta}(h(V)+\operatorname{deg} V)^{\operatorname{codim} B+\eta}\left[k_{\mathrm{tor}}(V): k_{\mathrm{tor}}\right]^{\operatorname{codim} B-1+\eta}, \\
\operatorname{deg} Y \ll_{\eta} \operatorname{deg} V\left((h(V)+\operatorname{deg} V)\left[k_{\mathrm{tor}}(V): k_{\mathrm{tor}}\right]\right)^{\operatorname{codim} B-1+\eta} .
\end{gathered}
$$

In addition, the torsion points $\zeta$ belong to a finite set of cardinality effectively bounded in terms of $\operatorname{deg} V, \operatorname{deg} B$ and constants depending only on $A$.

This theorem can be reformulated in the context of several other well-known conjectures, as explained in the introduction of [Checcoli et al. 2014].

The proof of Theorem 1.4 is split into two sections, depending on whether $Y$ is a translate or not: in Section 4 we prove part (1) and in Section 5 we prove parts (2) and (3).

The main ingredients (see Section 2.3) needed for the proof of Theorem 1.4 are Zhang's inequality, the arithmetic Bézout theorem by P. Philippon, our sharp Bogomolov-type bound proved in [Checcoli et al. 2012], and the relative Lehmer estimate by M. Carrizosa. As usual, the CM hypothesis is due to the use of a Lehmer bound, known only for CM varieties. This result is only needed when $Y$ is a translate, while case (1) of Theorem 1.4 holds with the weaker assumption that $A$ has a positive density of ordinary primes, as required to apply a Bogomolov-type bound (see [Galateau 2010, p. 779]). In particular, our method could treat the case of general abelian varieties, if the Lehmer- and Bogomolov-type bounds were known in such generality.

In Theorem 1.5, proved in Section 6, we expand our method in order to get some new effective results for curves in abelian varieties. This is particularly relevant, as bounds for the height in weak-transverse curves are hard to obtain. For instance, such bounds allow us to deduce some cases of the effective Mordell-Lang conjecture, stated in Corollary 1.6. The two classical approaches to the effective 
Mordell-Lang conjecture in abelian varieties are the Chabauty-Coleman and the Manin-Demjanenko methods. These methods require hypotheses that are similar to ours, but our result is of easier application and more explicit. Finally in Section 7, we give some generalisations to varieties in abelian varieties.

In particular we prove the following results. We fix an isogeny of the CM abelian variety $A$ to the product $\prod_{i=1}^{\ell} A_{i}^{e_{i}}$ of nonisogenous simple factors $A_{i}$ of dimension $g_{i}$. Since isogenies preserve finiteness, without loss of generality, we identify $A$ with $\prod_{i=1}^{\ell} A_{i}^{e_{i}}$. If $H \subseteq A$ is a subgroup, then $H=H_{1} \times \cdots \times H_{\ell}$, where $H_{i} \subseteq A_{i}^{e_{i}}$ is isogenous to $A_{i}^{f_{i}}$ for some $f_{i} \leq e_{i}$; therefore the matrix of the coefficients of the forms defining $H$ has the structure of a block diagonal matrix with entries in the endomorphism ring of the corresponding varieties. We can now state our effective result for weak-transverse curves, which is an example for the effective Zilber-Pink conjecture.

Theorem 1.5. Let $C \subseteq A=\prod_{i=1}^{\ell} A_{i}^{e_{i}}$ be a weak-transverse curve. Then the set

$$
\mathscr{S}(C)=C \cap\left(\bigcup_{H \in \mathscr{F}} H\right)
$$

is a set of bounded Néron-Tate height, where $\mathscr{F}$ is the family of all subgroups $H=\prod_{i=1}^{\ell} H_{i} \subseteq A$ such that

$$
\operatorname{codim} H_{j}>g_{j} \operatorname{dim} H_{j}
$$

for at least one index $j$ (here codim $H_{j}$ is the codimension of $H_{j}$ in $A_{j}^{e_{j}}$ ).

More precisely, if $Y \in C \cap H$, then for any real $\eta>0$, there exists a constant, depending only on $A$ and $\eta$, such that

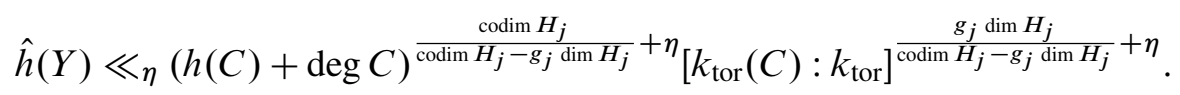

To prove Theorem 1.5 we first work in the projection on the $j$-th factor of $A$, and then we lift the construction to the variety $A$.

As an immediate consequence, we deduce the following corollary (proved in Section 6.1).

Let $\Gamma$ be a subgroup of $A=\prod_{i=1}^{\ell} A_{i}^{e_{i}}$. Assume that the group $\bar{\Gamma}_{i}<A_{i}$ generated by the coordinates of the projections of $\Gamma$ on the factors $A_{i}^{e_{i}}$ is an $\operatorname{End}\left(A_{i}\right)$-module of rank $t_{i}$.

Corollary 1.6. Let $A$ be a $C M$ abelian variety and let $C$ be a weak-transverse curve in $A$. Let $\Gamma$ be a subgroup as above, and suppose that $t_{j}<e_{j} /\left(g_{j}+1\right)$ for some index $j$. Then, for any positive $\eta$, there exists a constant depending only on $A$ 
and $\eta$, such that the set $C \cap \Gamma$ has Néron-Tate height bounded as

$$
\hat{h}(C \cap \Gamma) \ll_{\eta}(h(C)+\operatorname{deg} C)^{\frac{e_{j}-t_{j}}{e_{j}-\left(g_{j}+1\right) t_{j}}+\eta}\left[k_{\mathrm{tor}}(C): k_{\mathrm{tor}}\right]^{\frac{g_{j} t_{j}}{e_{j}-\left(g_{j}+1\right) t_{j}}}+\eta .
$$

We remark that the corollary applies also to some $\Gamma$ of infinite rank; indeed, we only assume that the rank on one projection is small (see Remark 6.1).

\section{Preliminaries}

2.1. Height and subgroups. We assume that all varieties are defined over the field of algebraic numbers.

Let $A$ be an abelian variety with CM. We fix, up to an isogeny, a decomposition of $A=\prod_{i=1}^{\ell} A_{i}^{e_{i}}$ in simple factors of dimension $\operatorname{dim} A_{i}=g_{i}$. We consider an embedding $i_{\mathscr{L}}$ of $A$ in $\mathbb{P}^{m}$ given by a symmetric ample line bundle $\mathscr{L}$ on $A$. Heights and degrees corresponding to $\mathscr{L}$ are computed via $i_{\mathscr{L}}$. More precisely, the degree of a subvariety of $A$ is the degree of its image under $i_{\mathscr{L}} ; \hat{h}=\hat{h}_{\mathscr{L}}$ is the $\mathscr{L}$-canonical Néron-Tate height of a point in $A$, and $h$ is the normalised height of a subvariety of $A$ as defined, for instance, in [Philippon 1991]. Notice that if $x \in A$ is a point, then $\hat{h}(x)=h(x)$.

By Lemma 2.2 in [Masser and Wüstholz 1993], if $A$ is an abelian variety defined over a number field $k$, then every abelian subvariety of $A$ is defined over a finite extension of $k$ of degree bounded by $3^{16(\operatorname{dim} A)^{4}}$; thus, without loss of generality, we assume that all abelian subvarieties of $A$ are defined over $k$.

Let $B+\zeta$ be an irreducible torsion variety of $A$. Then $B=B_{1} \times \cdots \times B_{l}$, where $B_{i} \subseteq A_{i}^{e_{i}}$ is isogenous to $A_{i}^{f_{i}}$ for some integer $0 \leq f_{i} \leq e_{i}$.

There exists a natural correspondence between abelian subvarieties $B$ of $A$, morphisms from $A$ to $\prod_{i=1}^{\ell} A_{i}^{e_{i}-f_{i}}$, and matrices made of $\ell$ blocks where the $i$-th block is an $\left(e_{i}-f_{i}\right) \times e_{i}$-matrix with entries in the endomorphism ring of $A_{i}$. For details on such a correspondence see, for instance, [Checcoli et al. 2012, Section 2.5]. In short, the abelian subvariety $B$ defines the projection morphism $\pi_{B}: A \rightarrow A / B$. The successive minima of $B$ give a matrix $\mathscr{H}_{B}$ of the above type. By multiplication on the left, the matrix $\mathscr{H}_{B}$ gives a morphism $\Phi_{B}$ from $A$ to $\prod_{i=1}^{\ell} A_{i}^{e_{i}-f_{i}}$, where $B$ is the zero component of $\operatorname{ker} \Phi_{B}$.

By Minkowski's theorem, $\operatorname{deg} B$ is (up to constants depending only on $A$ ) the product of the squares of the norms of the rows of $\mathscr{H}_{B}$. In addition, $B$ is the zero component of the zero set of the forms $h_{1}, \ldots, h_{r}$ corresponding to the rows of $\mathscr{H}_{\boldsymbol{B}}$. We order the $h_{i}$ by increasing degrees $d_{i}$ so that

$$
d_{1} \cdots d_{r} \ll \operatorname{deg}(B+\zeta) \ll d_{1} \cdots d_{r}
$$


We also recall that, from [Masser and Wüstholz 1993, Lemmas 1.3 and 1.4], if $B$ is an abelian subvariety of $A$ and $B^{\perp}$ is its orthogonal complement, then $\operatorname{deg} B^{\perp} \ll \operatorname{deg} B$, and therefore $\#\left(B \cap B^{\perp}\right) \ll(\operatorname{deg} B)^{2}$.

2.2. Torsion anomalous varieties. We recall some preliminary lemmas on torsion anomalous varieties used for our geometric constructions in the following sections.

Lemma 2.1 [Checcoli et al. 2014, Lemma 3.5]. Let $Y$ be a maximal $V$-torsion anomalous variety and let $B+\zeta$ be minimal for $Y$. Then $Y$ is weak-transverse in $B+\zeta$ (i.e., $Y$ is not contained in any proper torsion subvariety of $B+\zeta$ ).

Lemma 2.2 [Checcoli et al. 2014, Lemma 3.6]. Let $Y$ be a maximal $V$-torsion anomalous variety, and let $B+\zeta$ be minimal for $Y$. Then $Y$ is a component of $V \cap\left(B^{\prime}+\zeta\right)$ for every algebraic subgroup $B^{\prime} \supseteq B$ with $\operatorname{codim} B^{\prime} \geq \operatorname{dim} V-\operatorname{dim} Y$.

The following lemma is due to Philippon [2012] and to certain properties of orthogonality in the Mordell-Weil groups studied by D. Bertrand [1986].

We recall that the essential minimum of a subvariety $X \subseteq A$ is defined as

$$
\mu(X)=\sup \{\theta \in \mathbb{R} \mid\{x \in X(\overline{\mathbb{Q}}) \mid \hat{h}(x) \leq \theta\} \text { is nondense in } X\} .
$$

Lemma 2.3. Let $H+Y_{0}$ be a weak-transverse translate in $A$, with $Y_{0}$ a point in the orthogonal complement $H^{\perp}$ of $H$. Then $\mu\left(Y_{0}\right)=\mu\left(H+Y_{0}\right)$.

We conclude with a remark on translations by torsion points.

Remark 2.4. Notice that, for any subvariety $X$ of $A$, translations by a torsion point $\zeta$ leave invariant the degree, the field of definition over $k_{\text {tor }}$ and the normalised height of $X$ (see also [Philippon 1991, Proposition 9]). In addition, if $Y \subseteq V \cap(B+\zeta)$ is $V$-torsion anomalous, then $Y-\zeta \subseteq(V-\zeta) \cap B$ is $(V-\zeta)$-torsion anomalous. Therefore, without loss of generality, we can work in $V$ or in $V-\zeta$ with the advantage, in the latter case, that $B$ is an abelian subvariety.

2.3. Main ingredients. We recall here the main ingredients used in the proof of Theorem 1.4.

2.3.1. The Zhang estimate. The theorem below follows from the crucial result in Zhang's proof [1998] of the Bogomolov conjecture and from the definition of normalised height.

Theorem 2.5. Let $X \subseteq A$ be an irreducible subvariety.

Then

$$
\mu(X) \leq \frac{h(X)}{\operatorname{deg} X} \leq(1+\operatorname{dim} X) \mu(X) .
$$


2.3.2. The arithmetic Bézout theorem. The following version of the arithmetic Bézout theorem is due to Philippon [1995].

Theorem 2.6. Let $X$ and $Y$ be irreducible subvarieties of the projective space $\mathbb{P}^{n}$ defined over $\overline{\mathbb{Q}} ;$ let $Z_{1}, \ldots, Z_{g}$ be the irreducible components of $X \cap Y$. Then

$$
\sum_{i=1}^{g} h\left(Z_{i}\right) \leq \operatorname{deg} X h(Y)+\operatorname{deg} Y h(X)+c(n) \operatorname{deg} X \operatorname{deg} Y,
$$

where $c(n)$ is a constant depending only on $n$.

2.3.3. An effective Bogomolov estimate. The following sharp Bogomolov bound is proved by [Checcoli et al. 2012] and generalises a result of A. Galateau [2010].

Theorem 2.7 (Checcoli, Veneziano, Viada). Let $A$ be an abelian variety with a positive density of ordinary primes, and let $Y$ be an irreducible subvariety of $A$ transverse in a translate $B+p$. Then, for any $\eta>0$, there exists a positive constant $c_{1}$ depending on $A$ and $\eta$, such that

$$
\mu(Y) \geq c_{1} \frac{(\operatorname{deg} B)^{1 /(\operatorname{dim} B-\operatorname{dim} Y)-\eta}}{(\operatorname{deg} Y)^{1 /(\operatorname{dim} B-\operatorname{dim} Y)+\eta}} .
$$

2.3.4. A relative Lehmer estimate. The following Lehmer bound is proved in [Carrizosa 2009].

Theorem 2.8. Let $A$ be an abelian variety with $C M$ defined over a number field $k$, and let $k_{\mathrm{tor}}$ be the field of definition of all torsion points of $A$. Let $P$ be a point of infinite order in $A$, and let $B+\zeta$ be the torsion variety of minimal dimension containing $P$, with $B$ an abelian subvariety and $\zeta$ a torsion point. Then for every $\eta>0$, there exists a positive constant $c_{2}$ depending on $A$ and $\eta$, such that

$$
\hat{h}(P) \geq c_{2} \frac{(\operatorname{deg} B)^{1 / \operatorname{dim} B-\eta}}{\left[k_{\mathrm{tor}}(P): k_{\mathrm{tor}}\right]^{1 / \operatorname{dim} B+\eta}} .
$$

\section{Finitely many maximal $V$-torsion anomalous varieties in $V \cap\left(B+\operatorname{Tor}_{A}\right)$}

Let $V$ be a weak-transverse variety in an abelian variety $A$. Let us fix an abelian subvariety $B$ of $A$. In [Checcoli et al. 2014, Lemma 3.9] we proved that there are only finitely many $\zeta \in \operatorname{Tor}_{B^{\perp}}$ such that $V \cap(B+\zeta)$ has a maximal $V$-torsion anomalous component. In this section we prove that the number of such $\zeta$ is effectively bounded in terms of $\operatorname{deg} V, \operatorname{deg} B$ and some constants depending on $A$ (Proposition 3.5). We thank the referee for pointing out the effectivity question and for his useful comments.

The proof of such an effective result is based on an induction on the dimension of $V$, on Rémond's quantitative version of the Manin-Mumford conjecture [2000] 
and on the effective bound for the degree of the maximal translates in a variety implied, for instance, by a result of Bombieri and Zannier [1996]. We first recall these results and some other well-known bounds.

Recall that $A$ is an abelian variety and $\mathscr{L}$ is a symmetric ample line bundle on $A$. We denote by $h_{1}(A)$ the projective height of the zero of $A$ in the embedding associated with $\mathscr{L}^{\otimes 16}$ (as defined in [David and Philippon 2002, Notation 3.2]) and by $d_{A}$ the degree of the field of definition of $A$. If $G$ is an abelian subvariety of $A$ or a quotient of $A$, then $h_{1}(G)$ is bounded in terms of $h_{1}(A), \operatorname{deg} A, \operatorname{dim} A$ and $\operatorname{deg} G$ (see [ibid., Proposition 3.9]).

Moreover, in several works, Masser and Wüstholz and then other authors proved that for any abelian subvariety $G$ of $A$, the degree of the field of definition of $G$ is at most $3^{16(\operatorname{dim} A)^{4}} d_{A}$ (see [Masser and Wüstholz 1993, Lemma 2.2]). Below, we sum up these bounds.

Estimate 3.1. If $G$ is an abelian subvariety of $A$ or a quotient of $A$, then

- $d_{G}$ is bounded in terms of $d_{A}$ and $\operatorname{dim} A$;

- $h_{1}(G)$ is bounded in terms of $h_{1}(A), \operatorname{deg} A, \operatorname{dim} A$ and $\operatorname{deg} G$.

For simplicity, in what follows we shall denote by $c(A)$ any constant depending on $\operatorname{dim} A, d_{A}, h_{1}(A)$ and $\operatorname{deg} A$.

We recall the following consequence of Rémond's result [2000, Theorem 1.2].

Estimate 3.2. The number of irreducible components of the closure of the torsion of a weak-transverse variety $V$ in an abelian variety $A$ is effectively bounded as

$$
c(A)(\operatorname{deg} V)^{(\operatorname{dim} A)^{5(\operatorname{dim} V+1)^{2}}} .
$$

Following the work of Rémond [2000, Theorem 2.1] and the results in [David and Philippon 2002] one sees that if $G$ is an abelian subvariety of $A$ or a quotient of $A$, then the corresponding constant $c(G)$ appearing in Estimate 3.2 is bounded only in terms of $\operatorname{dim} A, d_{A}, h_{1}(A), \operatorname{deg} A$ and $\operatorname{deg} G$.

In our previous joint work with F. Veneziano [Checcoli et al. 2014, Lemma 7.4], we gave an explicit version of a corollary of Lemma 2 in [Bombieri and Zannier 1996]. This is a bound for the degree of the maximal translates contained in a variety, and so in particular for the degree of each component of the closure of the torsion. More precisely:

Estimate 3.3. If $V$ is weak-transverse in an abelian variety $A$, then the maximal translates contained in $V$ have degree bounded by $c(A)(\operatorname{deg} V)^{2^{\operatorname{dim} V}}$.

Notice that if $\zeta$ is a torsion point such that $V \cap(B+\zeta)$ has a $V$-torsion anomalous component, then all the points in $\zeta+\left(B \cap B^{\perp}\right)$ share the same property. Indeed $B+\zeta=B+\zeta+\left(B \cap B^{\perp}\right)$. Clearly, we shall avoid such a redundancy and work 
up to points in $B \cap B^{\perp}$. Nevertheless, $\left|B \cap B^{\perp}\right| \ll(\operatorname{deg} B)^{2}$. This makes the following definition consistent.

Definition 3.4. Let $B$ be an abelian subvariety of an abelian variety $A$. Let $V$ be a weak-transverse subvariety of $A$. We denote by $Z_{V, A}$ the set of torsion points $\zeta \in B^{\perp} / B \cap B^{\perp}$ such that $V \cap(B+\zeta)$ has a maximal $V$-torsion anomalous component $Y_{\zeta}$.

We point out that the set $Z_{V, A}$ also depends on $B$. However, in our proof $B$ is fixed, while $V$ and $A$ vary. To simplify the notation we only indicate the dependence on $V$ and $A$.

In the following proposition we estimate the number of points in $Z_{V, A}$. The number of maximal $V$-torsion anomalous components in $V \cap\left(B+\operatorname{Tor}_{A}\right)$ is clearly estimated by $\left|Z_{V, A}\right| \operatorname{deg} V \operatorname{deg} B$.

Proposition 3.5. Let $B$ be an abelian subvariety of an abelian variety $A$. Let $V$ be weak-transverse in $A$. Then the cardinality of $Z_{V, A}$ is effectively bounded in terms of $\operatorname{deg} V, \operatorname{deg} B$ and constants depending only on $\operatorname{dim} A, h_{1}(A), d_{A}$ and $\operatorname{deg} A$.

Proof. Consider the projection

$$
\pi_{B}: A \rightarrow A / B .
$$

We recall that the degree of the image via $\pi_{B}$ of a variety $X \subseteq A$ and the degree of the preimage via $\pi_{B}$ of a variety $X \subseteq A / B$ only depend on $\operatorname{deg} X, \operatorname{deg} B$ and $\operatorname{deg} A$. In particular, $\operatorname{deg} \pi_{B}(V)$ is bounded in terms of $\operatorname{deg} V, \operatorname{deg} B$, and $\operatorname{deg} A$ and $\operatorname{deg} A / B$ is bounded in terms of $\operatorname{deg} B$ and $\operatorname{deg} A$.

The proof of our proposition is done by induction on the dimension of $V$.

The base of our induction is the case of a curve, i.e., $\operatorname{dim} V=1$. Then $\pi_{B}(V)$ is a weak-transverse curve in $A / B$ because $V$ is weak-transverse in $A$. Moreover the points of $Z_{V, A}$ map to torsion points of $\pi_{B}(V)$. The number of torsion points of $\pi_{B}(V)$ is estimated using Estimate 3.2. Their preimage, which contains $Z_{V, A}$, then has cardinality effectively bounded in terms of $\operatorname{deg} V, \operatorname{deg} B$ and $c(A)$.

Suppose by inductive hypothesis that the proposition holds for every variety $V$ with $\operatorname{dim} V<n$. We then show that it holds for $V$ of dimension $n$.

To prove our result, we are going to partition $Z_{V, A}$ into a finite union of subsets $Z_{X}$ associated with irreducible subvarieties $X$ of $V$ of dimension $<n$. We then verify that such varieties $X$ satisfy the assumption of the proposition; by the inductive hypothesis, we deduce that the cardinalities $\left|Z_{X}\right|$ are effectively bounded in terms of $\operatorname{deg} V, \operatorname{deg} B$ and $c(A)$.

Denote by $f: V \rightarrow A / B$ the restriction of $\pi_{B}$ to $V$.

If $f$ is dominant, then the generic fibre $F_{p}=V \cap(B+\tilde{p})$ has dimension

$$
\operatorname{dim} F_{p}=\operatorname{dim} V-\operatorname{codim} B,
$$


where $p$ belongs to an open subset of $A / B$ and $f(\tilde{p})=p$. The dimensional equation (1) shows that the generic fibre is not anomalous. Consider the subset $V_{\pi}$ of $A / B$ given by all points that do not have generic fibre. By the fibre dimension theorem (see, for instance, [Shafarevich 1972, Section 6.3, Theorem 7]), this is a proper closed subset of $\pi_{B}(V)$ and its degree is effectively bounded in terms of $\operatorname{deg} V, \operatorname{deg} B$ and $c(A)$. Note that the image of $Z_{V, A}$ via $\pi_{B}$ is a subset of the torsion of $V_{\pi}$; indeed the fibre of a point in $\pi_{B}\left(Z_{V, A}\right)$ is torsion anomalous and therefore does not satisfy the equality (1).

If $f$ is not dominant, then set $V_{\pi}=\pi_{B}(V)$. Clearly, $Z_{V, A}$ is a subset of the torsion of $V_{\pi}$.

Note that in both cases

(a) $\operatorname{deg} V_{\pi}$ is bounded in terms of $\operatorname{deg} V, \operatorname{deg} B$ and $c(A)$.

Let $T_{1}, \ldots, T_{r}$ be the isolated components of the closure of the torsion of $V_{\pi}$ intersecting $\pi_{B}\left(Z_{V, A}\right)$. Clearly

and

$$
Z_{V, A}=\bigcup_{i=1}^{r}\left(\pi_{B}^{-1}\left(T_{i}\right) \cap Z_{V, A}\right)
$$

$$
\left|Z_{V, A}\right|=\sum_{i=1}^{r}\left|\pi_{B}^{-1}\left(T_{i}\right) \cap Z_{V, A}\right| .
$$

From Estimate 3.2 and (a), the number $r$ is effectively bounded in terms of deg $V$, $\operatorname{deg} B, \operatorname{deg} A$ and $c(A)$. Thus we shall prove that, for every $1 \leq i \leq r$, the cardinality $\left|\left(\pi_{B}^{-1}\left(T_{i}\right) \cap Z_{V, A}\right)\right|$ is effectively bounded in terms of $\operatorname{deg} V, \operatorname{deg} B$ and $c(A)$.

Let $T$ be one of the above components. Define

$$
W=\pi_{B}^{-1}(T) \cap V .
$$

We have that:

(i) $\operatorname{deg} W$ is bounded in terms of $\operatorname{deg} V, \operatorname{deg} B$ and $\operatorname{deg} A$. Indeed, by Bézout's theorem, $\operatorname{deg} W \leq \operatorname{deg} \pi_{B}^{-1}(T) \operatorname{deg} V$. By Estimate 3.3, $\operatorname{deg} T$ is bounded in terms of the degree and the dimension of $V_{\pi}$ and thus, by (a), in terms of $\operatorname{deg} B, \operatorname{deg} V$ and $c(A)$.

(ii) $\operatorname{dim} W<n$ because $V$ is weak-transverse in $A$ and so it is not contained in $\pi_{B}^{-1}(T)$.

(iii) For $\zeta \in \pi_{B}^{-1}(T) \cap Z_{V, A}$, each maximal $V$-torsion anomalous component $Y_{\zeta}$ of $V \cap(B+\zeta)$ is contained in $W$; indeed, $\pi_{B}\left(Y_{\zeta}\right)=\pi_{B}(\zeta) \in T$.

By (iii), the variety $W$ contains all the $Y_{\zeta}$ that we are counting; however, $W$ is not necessarily irreducible. Therefore we cannot hope to use the inductive hypothesis on $W$ and we have to consider its irreducible components. 
Let $X_{1}, \ldots, X_{S}$ be the irreducible components of $W$. For $\zeta \in \pi_{B}^{-1}(T) \cap Z_{V, A}$, we denote by $Y_{\zeta}$ any maximal $V$-torsion anomalous component of $V \cap(B+\zeta)$. By (iii), clearly each $Y_{\zeta}$ is contained in some $X_{i}$. We are going to count the number of $Y_{\zeta}$ contained in each $X_{i}$.

Denote

$$
Z_{X_{j}}=\left\{\zeta \in \pi_{B}^{-1}(T) \cap Z_{V, A} \mid X_{j} \text { contains some } Y_{\zeta}\right\} /\left(B \cap B^{\perp}\right) .
$$

Then

$$
\pi_{B}^{-1}(T) \cap Z_{V, A}=\bigcup_{j=1}^{s} Z_{X_{j}} .
$$

The number $s$ of irreducible components of $W$ is bounded by $\operatorname{deg} W$. Thus, by (i), $s$ is effectively bounded only in terms of $\operatorname{deg} V, \operatorname{deg} B$ and $c(A)$.

To conclude our proof we are left to bound in an effective way the cardinality of each $Z_{X}$ for $X$ running over all irreducible components of $W$.

If $X$ does not contain any $Y_{\zeta}$, then $\left|Z_{X}\right|=0$.

If $X=Y_{\zeta_{0}}$ for some $\zeta_{0} \in Z_{V, A}$, then $\left|Z_{X}\right|=1$.

Suppose that $X$ strictly contains $Y_{\zeta_{0}}$ for some $\zeta_{0} \in Z_{V, A}$. In this case we are going to show that $\left|Z_{X}\right| \leq\left|Z_{X-\zeta_{0}, \pi_{B}^{-1}(T)-\zeta_{0}}\right|$. Applying the inductive hypothesis, we estimate $\left|Z_{X-\zeta_{0}, \pi_{B}^{-1}(T)-\zeta_{0}}\right|$ in terms of $\operatorname{deg} V, \operatorname{deg} B$ and $c(A)$.

We first verify that $X-\zeta_{0}$ in $\pi_{B}^{-1}(T)-\zeta_{0}$ satisfies the assumption of the inductive hypothesis, that is, the assumption of the proposition with $\operatorname{dim} X<n$. Observe that we need to translate by $\zeta_{0}$ in order to obtain ambient varieties which are abelian varieties.

- The variety $\pi_{B}^{-1}(T)-\zeta_{0}$ is an abelian variety containing $B$. Indeed $B+\zeta_{0}$ is a subvariety of $\pi_{B}^{-1}(T)$, and $\zeta_{0} \in \pi_{B}^{-1}(T)$.

- The variety $X-\zeta_{0}$ is weak-transverse in $\pi_{B}^{-1}(T)-\zeta_{0}$. Equivalently, by Remark 2.4, we show that $X$ is weak-transverse in $\pi_{B}^{-1}(T)$. Since $Y_{\zeta_{0}}$ is a maximal $V$-torsion anomalous variety and $X$ strictly contains $Y_{\zeta_{0}}$, then $X$ cannot be $V$-torsion anomalous. Recall that $X$ is a component of $V \cap \pi_{B}^{-1}(T)$. Thus

$$
\operatorname{dim} \pi_{B}^{-1}(T)-\operatorname{dim} X=\operatorname{dim} A-\operatorname{dim} V .
$$

If $X$ was not weak-transverse in $\pi_{B}^{-1}(T)$, then $X \subseteq B_{1} \cap V$ with $B_{1} \subsetneq \pi_{B}^{-1}(T)$ a torsion variety. This contradicts relation (2).

- By (ii), $\operatorname{dim} X \leq \operatorname{dim} W<n$.

Thus, by inductive hypothesis, we get that $\left|Z_{X-\zeta_{0}, \pi_{B}^{-1}(T)-\zeta_{0}}\right|$ is effectively bounded in terms of $\operatorname{deg} X, \operatorname{deg} B, c\left(\pi_{B}^{-1}(T)\right)$. 
We now show that by our construction $\operatorname{deg} X$ and $c\left(\pi_{B}^{-1}(T)\right)$ only depend on $\operatorname{deg} V, \operatorname{deg} B$ and $A$.

- By (i), $\operatorname{deg} X \leq \operatorname{deg} W$ is effectively bounded in terms of $\operatorname{deg} V, \operatorname{deg} B$ and $c(A)$.

- By Estimate 3.2, we know that deg $T$ is effectively bounded in terms of $\operatorname{deg} V_{\pi}$ and $\operatorname{dim} V$. Moreover, by (a), $\operatorname{deg} V_{\pi}$ is bounded in terms of $\operatorname{deg} V, \operatorname{deg} B$ and $c(A)$. Finally, Estimate 3.1 ensures that $h_{1}\left(\pi_{B}^{-1}(T)\right)$ and $d_{\pi_{B}^{-1}(T)}$ are effectively bounded in terms of $\operatorname{deg} V, \operatorname{deg} B$ and $c(A)$.

Therefore,

$\left|Z_{X-\zeta_{0}, \pi_{B}^{-1}(T)-\zeta_{0}}\right|$ is effectively bounded in terms of $\operatorname{deg} V, \operatorname{deg} B$ and $c(A)$.

We finally prove that

$$
\left|Z_{X}\right| \leq\left|Z_{X-\zeta_{0}, \pi_{B}^{-1}(T)-\zeta_{0}}\right|
$$

We shall show that for every maximal $V$-torsion anomalous variety $Y_{\zeta} \subseteq X$, the variety $Y_{\zeta}-\zeta_{0}$ is a maximal $\left(X-\zeta_{0}\right)$-torsion anomalous variety in $\pi^{-1}(T)-\zeta_{0}$.

Clearly $Y_{\zeta}-\zeta_{0} \subseteq\left(X-\zeta_{0}\right) \cap\left(B+\zeta-\zeta_{0}\right)$. Since $Y_{\zeta}$ is $V$-torsion anomalous we have

$$
\operatorname{dim} B-\operatorname{dim} Y_{\zeta}<\operatorname{dim} A-\operatorname{dim} V .
$$

From this and (2) we obtain

$$
\operatorname{dim} B-\operatorname{dim} Y_{\zeta}<\operatorname{dim} A-\operatorname{dim} V=\operatorname{dim} \pi_{B}^{-1}(T)-\operatorname{dim} X .
$$

Thus $Y_{\zeta}-\zeta_{0}$ is a $\left(X-\zeta_{0}\right)$-torsion anomalous variety.

In addition, $Y_{\zeta}-\zeta_{0}$ is maximal: let $Y^{\prime} \supset Y_{\zeta}-\zeta_{0}$ be a maximal $\left(X-\zeta_{0}\right)$-torsion anomalous variety and let $B^{\prime}+\zeta^{\prime}$ be minimal for $Y^{\prime}$. From (2), we have

$$
\operatorname{dim} B^{\prime}-\operatorname{dim} Y^{\prime}<\operatorname{dim} \pi_{B}^{-1}(T)-\operatorname{dim} X=\operatorname{dim} A-\operatorname{dim} V .
$$

Thus $Y^{\prime}+\zeta_{0} \subseteq V \cap\left(B^{\prime}+\zeta^{\prime}+\zeta_{0}\right)$ is $V$-torsion anomalous and contains $Y_{\zeta}$. The maximality of $Y_{\zeta}$ as $V$-torsion anomalous implies $Y^{\prime}+\zeta_{0}=Y_{\zeta}$.

In conclusion, collecting all our bounds, we have proven that $\left|Z_{V, A}\right|$ is effectively bounded in terms of $\operatorname{deg} V, \operatorname{deg} B$ and $c(A)$.

\section{Nontranslate torsion anomalous varieties}

Proof of Theorem 1.4, part (1). Let $Y$ be a maximal $V$-torsion anomalous variety which is not a translate, and so of positive dimension. Let $B+\zeta$ be minimal for $Y$. We use the arithmetic Bézout theorem and the Bogomolov bound to prove that deg $B$ is bounded only in terms of $V$ and $A$, then we deduce the bounds for $h(Y)$ and $\operatorname{deg} Y$. 
By Lemma 2.1, $Y$ is weak-transverse in $B+\zeta$, and by assumption $\operatorname{dim} B=$ $\operatorname{dim} Y+1$; therefore, $Y$ is transverse in $B+\zeta$. Applying the Bogomolov estimate (Theorem 2.7) to $Y$ in $B+\zeta$, we get

$$
\frac{(\operatorname{deg} B)^{1-\eta}}{(\operatorname{deg} Y)^{1+\eta}} \ll_{\eta} \mu(Y) .
$$

Let $h_{1}, \ldots, h_{r}$ be the forms of increasing degrees $d_{i}$ such that $B+\zeta$ is a component of their zero set. We have that $r \leq \operatorname{codim} B \leq r g$ and

$$
d_{1} \cdots d_{r} \ll \operatorname{deg}(B+\zeta)=\operatorname{deg} B \ll d_{1} \cdots d_{r} .
$$

Consider the algebraic subgroup given by the first $h_{1} \cdots h_{r-1}$ forms, and let $B^{\prime}$ be one of its irreducible components containing $B+\zeta$. Then by (4) we have

$$
\operatorname{deg} B^{\prime} \ll d_{1} \cdots d_{r-1} \ll(\operatorname{deg} B)^{(r-1) / r}
$$

and $\operatorname{codim} B^{\prime} \geq \operatorname{codim} B-g$.

Since codim $V \geq g+1=g+\operatorname{dim} B-\operatorname{dim} Y$, this implies that $\operatorname{codim} B^{\prime} \geq$ $\operatorname{dim} V-\operatorname{dim} Y$, and thus, by Lemma 2.2, $Y$ is a component of $V \cap B^{\prime}$.

We apply the arithmetic Bézout theorem to $V \cap B^{\prime}$ and recall that $h\left(B^{\prime}\right)=0$ because $B^{\prime}$ is a torsion variety; we get

$$
h(Y) \ll(h(V)+\operatorname{deg} V) \operatorname{deg} B^{\prime} \ll(h(V)+\operatorname{deg} V)(\operatorname{deg} B)^{(r-1) / r} .
$$

Zhang's inequality, with (3) and (5), gives

$$
\frac{(\operatorname{deg} B)^{1-\eta}}{(\operatorname{deg} Y)^{1+\eta}} \ll_{\eta} \mu(Y) \ll(h(V)+\operatorname{deg} V) \frac{(\operatorname{deg} B)^{(r-1) / r}}{\operatorname{deg} Y} .
$$

Recall that $Y$ is a component of $V \cap(B+\zeta)$. By Bézout's theorem, $\operatorname{deg} Y \leq$ $\operatorname{deg} B \operatorname{deg} V$, thus

$$
(\operatorname{deg} B)^{1-\eta} \ll_{\eta}(h(V)+\operatorname{deg} V)(\operatorname{deg} B)^{(r-1) / r}(\operatorname{deg} B \operatorname{deg} V)^{\eta},
$$

and therefore

$$
(\operatorname{deg} B)^{1 / r-2 \eta} \ll_{\eta}(h(V)+\operatorname{deg} V)(\operatorname{deg} V)^{\eta} .
$$

For $\eta$ small enough, we get

$$
\operatorname{deg} B \ll_{\eta}(h(V)+\operatorname{deg} V)^{r+\eta}(\operatorname{deg} V)^{\eta} ;
$$

this proves that the degree of $B$ is bounded only in terms of $V$ and $A$. Since there are finitely many abelian subvarieties of bounded degree, applying Proposition 3.5, we conclude that $\zeta$ belongs to a finite set of cardinality effectively bounded. 
The bound on the height of $Y$ is now given by (5) and (6):

$$
h(Y) \ll_{\eta}(h(V)+\operatorname{deg} V)^{r+\eta}(\operatorname{deg} V)^{\eta} .
$$

Finally, the bound on the degree is obtained from (6) and Bézout's theorem for the component $Y$ of $V \cap B^{\prime}$ :

$$
\operatorname{deg} Y \ll_{\eta}(h(V)+\operatorname{deg} V)^{r-1+\eta}(\operatorname{deg} V) .
$$

\section{Torsion anomalous translates}

Proof of Theorem 1.4, parts (2) and (3). Let $Y$ be a maximal $V$-torsion anomalous translate with $B+\zeta$ minimal for $Y$.

We proceed to bound $\operatorname{deg} B$ and, in turn, the height and the degree of $Y$, using the Lehmer estimate and the arithmetic Bézout theorem.

The variety $B+\zeta$ is a component of the torsion variety defined as the zero set of forms $h_{1}, \ldots, h_{r}$ of increasing degrees $d_{i}$, and

$$
d_{1} \cdots d_{r} \ll \operatorname{deg} B=\operatorname{deg}(B+\zeta) \ll d_{1} \cdots d_{r} .
$$

We have that $r \leq \operatorname{codim} B \leq r g$.

Consider the torsion variety defined as the zero set of the first $r-1$ forms $h_{1}, \ldots, h_{r-1}$, and take a connected component $B^{\prime}$ containing $B+\zeta$, so that $\operatorname{deg} B^{\prime} \ll d_{1} \cdots d_{r-1} \ll(\operatorname{deg} B)^{(r-1) / r}$ and $\operatorname{codim} B^{\prime} \geq \operatorname{codim} B-g$.

By Lemma 2.2, $Y$ is a component of $V \cap B^{\prime}$; indeed

$$
\operatorname{codim} B^{\prime} \geq \operatorname{codim} B-g=\operatorname{dim} A-g-\operatorname{dim} Y-1>\operatorname{dim} V-\operatorname{dim} Y-1 .
$$

The proof is now divided in two cases, depending on $\operatorname{dim} Y$. If $Y$ has dimension zero we use the arithmetic Bézout theorem and the Lehmer estimate; if $Y=H+Y_{0}$ is a translate of positive dimension, we can reduce to the zero dimensional case using some properties of the essential minimum.

Proof of part (2). Consider first the case of a maximal torsion anomalous point $Y$.

All conjugates of $Y$ over $k_{\text {tor }}(V)$ are components of $V \cap(B+\zeta)$; they all have the same normalised height and their number is at least

$$
\left[k_{\mathrm{tor}}(V, Y): k_{\mathrm{tor}}(V)\right] \geq \frac{\left[k_{\mathrm{tor}}(Y): k_{\mathrm{tor}}\right]}{\left[k_{\mathrm{tor}}(V): k_{\mathrm{tor}}\right]} .
$$

We then apply the arithmetic Bézout theorem in $V \cap B^{\prime}$, obtaining

$$
\left[k_{\text {tor }}(Y): k_{\text {tor }}\right] \hat{h}(Y) \ll(h(V)+\operatorname{deg} V)\left[k_{\text {tor }}(V): k_{\text {tor }}\right](\operatorname{deg} B)^{(r-1) / r} .
$$


Applying Theorem 2.8 to $Y$ in $B+\zeta$, we obtain that, for every positive real $\eta$,

$$
\hat{h}(Y) \gg_{\eta} \frac{(\operatorname{deg} B)^{1-\eta}}{\left[k_{\mathrm{tor}}(Y): k_{\mathrm{tor}}\right]^{1+\eta}} .
$$

Combining (8) and (7), we have

$$
\begin{aligned}
\frac{(\operatorname{deg} B)^{1-\eta}}{\left[k_{\mathrm{tor}}(Y): k_{\mathrm{tor}}\right]^{\eta}} & \ll_{\eta}\left[k_{\mathrm{tor}}(Y): k_{\mathrm{tor}}\right] \hat{h}(Y) \\
& \ll(h(V)+\operatorname{deg} V)\left[k_{\mathrm{tor}}(V): k_{\mathrm{tor}}\right](\operatorname{deg} B)^{(r-1) / r} .
\end{aligned}
$$

For $\eta$ small enough, we obtain

$$
\operatorname{deg} B \ll_{\eta}\left((h(V)+\operatorname{deg} V)\left[k_{\mathrm{tor}}(V): k_{\mathrm{tor}}\right]\right)^{r+\eta}\left[k_{\mathrm{tor}}(Y): k_{\mathrm{tor}}\right]^{\eta} .
$$

Apply now Bézout's theorem to $V \cap B^{\prime}$. All the conjugates of $Y$ over $k_{\text {tor }}(V)$ are components of this intersection, so

$$
\frac{\left[k_{\text {tor }}(Y): k_{\text {tor }}\right]}{\left[k_{\text {tor }}(V): k_{\text {tor }}\right]} \ll_{\eta}(\operatorname{deg} B)^{(r-1) / r}(\operatorname{deg} V) .
$$

Substituting (9) into (10) we have the last bound of part (2) in the statement.

Finally, we apply the arithmetic Bézout theorem to $V \cap B^{\prime}$ to get

$$
\begin{aligned}
\hat{h}(Y) & \ll(h(V)+\operatorname{deg} V)(\operatorname{deg} B)^{(r-1) / r} \\
& \ll_{\eta}(h(V)+\operatorname{deg} V)^{r+\eta}\left[k_{\mathrm{tor}}(V): k_{\mathrm{tor}}\right]^{r-1+\eta} .
\end{aligned}
$$

Having bounded $\operatorname{deg} B$, in view of Proposition 3.5 the points $\zeta$ belong to a finite set of cardinality effectively bounded.

Proof of part (3). Assume now that $Y$ is a translate of positive dimension and write $Y=H+Y_{0}$, with $H$ an abelian variety and $Y_{0}$ a point in $H^{\perp}$.

To bound $\operatorname{deg} B$ we can assume, without loss of generality, that $\zeta=0$ (see Remark 2.4). By Lemma 2.3,

$$
\mu\left(Y_{0}\right)=\mu\left(H+Y_{0}\right) .
$$

Since the intersection $V \cap B^{\prime}$ is defined over $k_{\text {tor }}(V)$, every conjugate of $H+Y_{0}$ over $k_{\text {tor }}(V)$ is a component of $V \cap B^{\prime}$; as before, such components have the same normalised height and their number is at least

$$
\frac{\left[k_{\mathrm{tor}}\left(H+Y_{0}\right): k_{\mathrm{tor}}\right]}{\left[k_{\mathrm{tor}}(V): k_{\mathrm{tor}}\right]} \text {. }
$$

We apply the arithmetic Bézout theorem in $V \cap B^{\prime}$ and we obtain

$$
h\left(H+Y_{0}\right) \frac{\left[k_{\text {tor }}\left(H+Y_{0}\right): k_{\text {tor }}\right]}{\left[k_{\text {tor }}(V): k_{\text {tor }}\right]} \ll(h(V)+\operatorname{deg} V)(\operatorname{deg} B)^{(r-1) / r} .
$$


By Zhang's inequality, (11) and (12), we deduce

$$
\mu\left(Y_{0}\right) \ll \frac{(h(V)+\operatorname{deg} V)\left[k_{\mathrm{tor}}(V): k_{\mathrm{tor}}\right](\operatorname{deg} B)^{(r-1) / r}}{\left[k_{\mathrm{tor}}\left(H+Y_{0}\right): k_{\mathrm{tor}}\right] \operatorname{deg} H} .
$$

The lower bound for $\mu\left(Y_{0}\right)$ is derived as in the case of dimension zero.

Consider the smallest abelian subvariety $H_{0}$ of $B$ containing $Y_{0}$. Clearly $H_{0}$ is the irreducible component of $H^{\perp} \cap B$ containing $Y_{0}$. Indeed, they are both one-dimensional abelian varieties containing the point $Y_{0}$ of infinite order.

By the definition of $H_{0}$, we have $B=H+H_{0}$, and from [Masser and Wüstholz 1993, Lemma 1.2], we obtain

$$
\#\left(H \cap H_{0}\right) \operatorname{deg} B \leq \operatorname{deg} H \operatorname{deg} H_{0} .
$$

Moreover, from $H \cap H_{0} \subseteq H \cap H^{\perp}$, we get

$$
\#\left(H \cap H_{0}\right) \leq \#\left(H \cap H^{\perp}\right) \ll(\operatorname{deg} H)^{2} .
$$

Applying Theorem 2.8 to $Y_{0}$ in $H_{0}$ we get that, for every positive real $\eta$,

$$
\mu\left(Y_{0}\right)=\hat{h}\left(Y_{0}\right) \gg_{\eta} \frac{\left(\operatorname{deg} H_{0}\right)^{1-\eta}}{\left[k_{\mathrm{tor}}\left(Y_{0}\right): k_{\mathrm{tor}}\right]^{1+\eta}} .
$$

We remark that

$$
\left[k_{\text {tor }}\left(Y_{0}\right): k_{\text {tor }}\right] \leq\left[k_{\text {tor }}\left(H+Y_{0}\right): k_{\text {tor }}\right] \cdot \#\left(H \cap H_{0}\right)
$$

because if $\sigma$ is in $\operatorname{Gal}\left(\bar{k}_{\mathrm{tor}} / k_{\mathrm{tor}}\left(H+Y_{0}\right)\right)$, then $\sigma\left(Y_{0}\right)-Y_{0}$ is in $H \cap H_{0}$, so $\left[k_{\text {tor }}\left(Y_{0}\right): k_{\text {tor }}\left(H+Y_{0}\right)\right] \leq \#\left(H \cap H_{0}\right)$.

Combining the upper bound and the lower bound for $\mu\left(Y_{0}\right)$ in (13) and (16), and using also (14), (15) and (17), for $\eta$ sufficiently small, we have

$$
\operatorname{deg} B \ll_{\eta}\left((h(V)+\operatorname{deg} V)\left[k_{\mathrm{tor}}(V): k_{\mathrm{tor}}\right]\right)^{r+\eta},
$$

where the dependence on deg $H\left[k_{\text {tor }}\left(H+Y_{0}\right): k_{\text {tor }}\right]$ has been removed by applying Bézout's theorem to the intersection $V \cap B^{\prime}$.

This also gives

$$
\operatorname{deg}\left(H+Y_{0}\right) \ll_{\eta}(\operatorname{deg} V)\left((h(V)+\operatorname{deg} V)\left[k_{\text {tor }}(V): k_{\text {tor }}\right]\right)^{r-1+\eta} .
$$

Finally, from (12), (18) and the trivial bound $\left[k_{\mathrm{tor}}\left(H+Y_{0}\right): k_{\mathrm{tor}}\right] \geq 1$, we obtain

$$
h\left(H+Y_{0}\right) \ll_{\eta}(h(V)+\operatorname{deg} V)^{r+\eta}\left[k_{\mathrm{tor}}(V): k_{\mathrm{tor}}\right]^{r-1+\eta} .
$$

Since we have bounded $\operatorname{deg} B$, we can conclude from Proposition 3.5 that the points $\zeta$ belong to a finite set of cardinality effectively bounded. 


\section{The case of a curve and applications to the effective Mordell-Lang conjecture}

Recall that $A=\prod_{i=1}^{\ell} A_{i}^{e_{i}}$ with $A_{i}$ nonisogenous simple CM factors of dimension $g_{i}$. To prove Theorem 1.5 we essentially follow the proof of Theorem 1.4, part (2), working first in the projection on one factor, and then lifting the construction to the abelian variety $A$.

Proof of Theorem 1.5. Clearly all the points in $\mathscr{S}(C)$ are $C$-torsion anomalous; in addition, since $C$ is a weak-transverse curve, each torsion anomalous point is maximal.

If $Y \in \mathscr{Y}(C)$, then $Y \in C \cap H$, with $H=\prod_{i} H_{i}$ the subgroup containing $Y$ which is minimal with respect to the inclusion.

Denote by $Y_{i}$ the projection of $Y$ on $H_{i}$ and by $C_{i}$ the projection of $C$ on $A_{i}^{e_{i}}$. Let $j$ be one of the indices satisfying the hypothesis of the theorem. Assume first that $Y_{j}$ is a torsion point, and define $H^{\prime}=A_{1}^{e_{1}} \times \cdots \times\left\{Y_{j}\right\} \times \cdots \times A_{\ell}^{e_{\ell}}$. Clearly $\operatorname{deg} H^{\prime} \ll 1$ and $h\left(H^{\prime}\right)=0$. Then, applying the arithmetic Bézout theorem to $Y$ in $C \cap H^{\prime}$, we get $\hat{h}(Y) \ll(h(C)+\operatorname{deg} C)$.

Assume now that $Y_{j}$ is not a torsion point. Let $B_{j}+\zeta_{j}$ be a component of $H_{j}$ containing $Y_{j}$. Clearly $\operatorname{dim} B_{j}=\operatorname{dim} H_{j}$ and $Y_{j} \in C_{j} \cap\left(B_{j}+\zeta_{j}\right)$ with $B_{j}+\zeta_{j}$ minimal for $Y_{j}$. Furthermore, $Y_{j}$ is a component of $C_{j} \cap\left(B_{j}+\zeta_{j}\right)$ because $C_{j}$ is weak-transverse and, by assumption, codim $H_{j}>g_{j} \operatorname{dim} H_{j}>0$. This ensures that the matrix associated to $B_{j}+\zeta_{j}$ has at least two rows, which is necessary to apply the method.

We now sketch the proof, which follows the proof of Theorem 1.4, part (2), and we give the relevant bounds.

The variety $B_{j}+\zeta_{j}$ is a component of the zero set of forms $h_{1}, \ldots, h_{r}$ of increasing degrees $d_{j}$ with

$$
d_{1} \cdots d_{r} \ll \operatorname{deg} B_{j}=\operatorname{deg}\left(B_{j}+\zeta_{j}\right) \ll d_{1} \cdots d_{r},
$$

and we have that $r=\operatorname{codim} B_{j} / g_{j}=\operatorname{codim} H_{j} / g_{j}$.

Consider the torsion variety defined as the zero set of $h_{1}$, and let $B_{j}^{\prime}$ be one of its connected components containing $B_{j}+\zeta_{j}$; then $\operatorname{deg} B_{j}^{\prime} \ll d_{1} \ll\left(\operatorname{deg} B_{j}\right)^{1 / r}=$ $\left(\operatorname{deg} B_{j}\right)^{g_{j} / \operatorname{codim} B_{j}}$.

From Theorem 2.8 applied to $Y_{j}$ in $B_{j}+\zeta_{j}$, for every positive real $\eta$, we get

$$
\hat{h}\left(Y_{j}\right) \gg_{\eta} \frac{\left(\operatorname{deg} B_{j}\right)^{1 / \operatorname{dim} B_{j}-\eta}}{\left[k_{\mathrm{tor}}\left(Y_{j}\right): k_{\mathrm{tor}}\right]^{1 / \operatorname{dim} B_{j}+\eta}} .
$$

Notice that all conjugates of $Y_{j}$ over $k_{\text {tor }}\left(C_{j}\right)$ are components of $C_{j} \cap B_{j}^{\prime}$ and they all have the same height. Applying the arithmetic Bézout theorem to $C_{j} \cap B_{j}^{\prime}$ 
and arguing as in the proof of Theorem 1.4, we have

$$
\hat{h}\left(Y_{j}\right) \frac{\left[k_{\mathrm{tor}}\left(Y_{j}\right): k_{\mathrm{tor}}\right]}{\left[k_{\mathrm{tor}}\left(C_{j}\right): k_{\mathrm{tor}}\right]} \ll\left(h\left(C_{j}\right)+\operatorname{deg} C_{j}\right)\left(\operatorname{deg} B_{j}\right)^{g_{j} / \operatorname{dim} B_{j}} .
$$

Recall that $\left[k_{\text {tor }}\left(Y_{j}\right): k_{\text {tor }}\right] \geq 1$. From (19) and (20) we get

$\left(\operatorname{deg} B_{j}\right)^{\frac{\operatorname{codim} B_{j}-g_{j} \operatorname{dim} B_{j}}{\operatorname{codim} B_{j} \operatorname{dim} B_{j}}-\eta}$

$$
\ll_{\eta}\left[k_{\mathrm{tor}}\left(C_{j}\right): k_{\mathrm{tor}}\right]\left(h\left(C_{j}\right)+\operatorname{deg} C_{j}\right)\left[k_{\mathrm{tor}}\left(Y_{j}\right): k_{\mathrm{tor}}\right]^{1 / \operatorname{dim} B_{j}-1+\eta} .
$$

Since codim $B_{j}-g_{j} \operatorname{dim} B_{j}=\operatorname{codim} H_{j}-g_{j} \operatorname{dim} H_{j} \geq 1$, for $\eta$ sufficiently small this yields

$$
\operatorname{deg} B_{j} \ll_{\eta}\left(\left[k_{\mathrm{tor}}\left(C_{j}\right): k_{\mathrm{tor}}\right]\left(h\left(C_{j}\right)+\operatorname{deg} C_{j}\right)\right)^{\frac{\operatorname{codim} H_{j} \operatorname{dim} H_{j}}{\operatorname{codim} H_{j}-g_{j} \operatorname{dim} H_{j}}+\eta},
$$

where if $\operatorname{dim} B_{j}>1$, we use $\left[k_{\mathrm{tor}}\left(Y_{j}\right): k_{\mathrm{tor}}\right] \geq 1$, and if $\operatorname{dim} B_{j}=1$, we use $\left[k_{\text {tor }}\left(Y_{j}\right): k_{\text {tor }}\right] \leq\left[k_{\text {tor }}\left(C_{j}\right): k_{\text {tor }}\right] \operatorname{deg} B \operatorname{deg} C_{j}$.

We now lift the construction to $A$ as follows. Define $H^{\prime}=A_{1}^{e_{1}} \times \cdots \times B_{j}^{\prime} \times \cdots \times A_{\ell}^{e_{\ell}}$. Clearly $\operatorname{deg} H^{\prime} \leq \operatorname{deg} A \operatorname{deg} B_{j}^{\prime}$ and $Y$ is a component of $C \cap H^{\prime}$. Applying the arithmetic Bézout theorem to $C \cap H^{\prime}$ and using (21), we obtain

$$
\begin{aligned}
& \hat{h}(Y) \ll(h(C)+\operatorname{deg} C) \operatorname{deg} H^{\prime} \ll(h(C)+\operatorname{deg} C)\left(\operatorname{deg} B_{j}\right)^{g_{j} / \operatorname{dim} H_{j}} \\
& \ll_{\eta}(h(C)+\operatorname{deg} C)^{\frac{\operatorname{codim} H_{j}}{\operatorname{codim} H_{j}-g_{j} \operatorname{dim} H_{j}}+\eta}\left[k_{\text {tor }}(C): k_{\text {tor }}\right]^{\frac{g_{j} \operatorname{dim} H_{j}}{\operatorname{codim} H_{j}-g_{j} \operatorname{dim} H_{j}}+\eta} \text {. }
\end{aligned}
$$

\subsection{An application to the effective Mordell-Lang conjecture.}

Proof of Corollary 1.6. Let $x \in C \cap \Gamma$. Let $j$ be an index such that $e_{j} /\left(g_{j}+1\right)>t_{j}$ and denote by $\left(x_{1}, \ldots, x_{e_{j}}\right)$ the projection of $x$ in $\Gamma_{j}$.

Let $\gamma_{1}, \ldots, \gamma_{t_{j}}$ be generators of the free part of $\bar{\Gamma}_{j}$. Then there exist elements $0 \neq a_{k} \in \operatorname{End}\left(A_{j}\right)$ for $k=1, \ldots, e_{j}$, an $e_{j} \times t_{j}$-matrix $M_{j}$ with coefficients in $\operatorname{End}\left(A_{j}\right)$ and a torsion point $\zeta \in A_{j}^{e_{j}}$ such that

$$
\left(a_{1} x_{1}, \ldots, a_{e_{j}} x_{e_{j}}\right)^{t}=M_{j}\left(\gamma_{1}, \ldots, \gamma_{t_{j}}\right)^{t}+\zeta .
$$

If the rank of $M_{j}$ is zero, then $\left(x_{1}, \ldots, x_{e_{j}}\right)$ is a torsion point and so has height zero.

If $M_{j}$ has positive rank $r_{j}$, we can choose $r_{j}$ equations of the system corresponding to $r_{j}$ linearly independent rows of $M_{j}$. We use these equations to write the $\gamma_{k}$ in terms of the $x_{k}$ and we substitute these expressions in the remaining equations. We obtain a system of maximal rank with $e_{j}-r_{j} \geq e_{j}-t_{j}$ linearly independent 
equations in the variables $x_{1}, \ldots, x_{e_{j}}$ :

$$
\left\{\begin{array}{l}
m_{11} x_{1}+\cdots+m_{1 e_{j}} x_{e_{j}}=\xi_{1}, \\
\vdots \\
m_{e_{j}-r_{j}, 1} x_{1}+\cdots+m_{e_{j}-r_{j}, e_{j}} x_{e_{j}}=\xi_{e_{j}-r_{j}},
\end{array}\right.
$$

where $\xi_{k} \in A_{j}$ are torsion points and $m_{k \ell} \in \operatorname{End}\left(A_{j}\right)$. These equations define a torsion variety $H_{j} \subseteq A_{j}^{e_{j}}$. Since $\left(g_{j}+1\right) t_{j}<e_{j}$ we have codim $H_{j}>g_{j} \operatorname{dim} H_{j}$.

Then $x \in C \cap H$, where $H$ satisfies the hypothesis of Theorem 1.5, which gives the bound for the height of $x$.

Remark 6.1. Notice that it is possible to apply the corollary also to subgroups $\Gamma$ whose rank is bounded only on one projection.

For example, let $E_{1}, E_{2}$ be two elliptic curves defined over $\mathbb{Q}$ and such that $E_{1}(\mathbb{Q})$ is an abelian group of rank 1 , and consider the product $A=E_{1}^{4} \times E_{2}$.

Let $C$ be a weak-transverse curve in $A$. Consider the subgroup $\Gamma=E_{1}(\mathbb{Q})^{4} \times$ $E_{2}(\overline{\mathbb{Q}})$ of $A$. Then $\Gamma$ is not of finite rank, but with the notation of the corollary, we have $g_{1}=1, e_{1}=4, t_{1}=1$ and $t_{1}<e_{1} /\left(g_{1}+1\right)=\frac{4}{2}$.

The hypothesis of the corollary is therefore verified, and we have that

$$
\hat{h}(C \cap \Gamma) \ll_{\eta}(h(C)+\operatorname{deg} C)^{3 / 2+\eta}\left[k_{\text {tor }}(C): k_{\text {tor }}\right]^{1 / 2+\eta} .
$$

\section{From curves to varieties}

We now adapt the proof strategy of Theorem 1.4 to obtain some new results for varieties $V$ of dimension $>1$ embedded in a power $E^{n}$ of a CM elliptic curve. For subvarieties of general $\mathrm{CM}$ abelian varieties some technical conditions arise. This makes a straightforward generalisation of our method of little interest.

For torsion anomalous varieties which are translates, the proof can be easily adapted, while for nontranslates a new argument is needed. Indeed, in this last case, the torsion anomalous variety is not transverse, but only weak-transverse in its minimal variety, a condition which is not sufficient to use the sharp Bogomolov bound.

The torsion varieties contained in $V$ are already covered by the Manin-Mumford conjecture, therefore we restrict ourselves to the $V$-torsion anomalous varieties which are not torsion.

Theorem 7.1. Let $E$ be a CM elliptic curve defined over a number field $k$ and let $n>1$ be an integer. Denote by $k_{\mathrm{tor}}$ a field of definition of all torsion points of $E$.

Let $V \subseteq E^{n}$ be a weak-transverse variety. Let $Y \subseteq V \cap B+\zeta$ be a maximal $V$-torsion anomalous variety which is not a torsion variety, and let $B+\zeta$ be minimal for $Y$.

Set $b=\operatorname{dim} B, v=\operatorname{dim} V$ and $y=\operatorname{dim} Y$ and assume that $(n-b)>(v-y)(b-y)$. 
Then for any $\eta>0$, there exist constants depending only on $E^{n}$ and $\eta$ such that:

(1) If $Y$ is a point, then

$$
\begin{gathered}
\operatorname{deg} B \ll_{\eta}\left((h(V)+\operatorname{deg} V)\left[k_{\mathrm{tor}}(V): k_{\mathrm{tor}}\right]\right)^{\frac{(n-b) b}{(n-b)-v b}+\eta}, \\
\hat{h}(Y) \ll \eta(h(V)+\operatorname{deg} V)^{\frac{n-b}{(n-b)-v b}+\eta}\left[k_{\mathrm{tor}}(V): k_{\mathrm{tor}}\right]^{\frac{v b}{(n-b)-v b}}+\eta \\
{\left[k_{\mathrm{tor}}(Y): k_{\mathrm{tor}}\right] \ll_{\eta} \operatorname{deg} V(h(V)+\operatorname{deg} V)^{\frac{v b}{(n-b)-v b}+\eta}\left[k_{\mathrm{tor}}(V): k_{\mathrm{tor}}\right]^{\frac{n-b}{(n-b)-v b}}+\eta}
\end{gathered}
$$

(2) If $Y$ is a translate of positive dimension, then

$\operatorname{deg} B \ll_{\eta}\left((h(V)+\operatorname{deg} V)\left[k_{\text {tor }}(V): k_{\text {tor }}\right]\right)^{\frac{(n-b)(b-y)}{(n-b)-(v-y)(b-y)}+\eta}$,

$h(Y) \ll_{\eta}(h(V)+\operatorname{deg} V)^{\frac{n-b}{(n-b)-(v-y)(b-y)}+\eta}\left[k_{\text {tor }}(V): k_{\text {tor }}\right]^{\frac{(v-y)(b-y)}{(n-(v-y)(b-y)}+\eta}$,

$\operatorname{deg} Y \ll_{\eta} \operatorname{deg} V\left((h(V)+\operatorname{deg} V)\left[k_{\text {tor }}(V): k_{\text {tor }}\right]\right)^{\frac{(v-y)(b-y)}{(n-b)-(v-y)}+\eta}$.

(3) If $Y$ is not a translate, then

$\operatorname{deg} B \ll_{\eta}\left((h(V)+\operatorname{deg} V)\left[k_{\text {tor }}(V): k_{\text {tor }}\right]\right)^{\frac{(b-y)(n-b)}{(n-b)-(v-y)(b-y)}}+\eta$,

$\left.h(Y) \ll \eta(h(V)+\operatorname{deg} V)^{\frac{(n-b)-(v-y)(b-y)}{(n-b)}}+k_{\text {tor }}(V): k_{\text {tor }}\right]^{\frac{(v-y)(b-y)}{(n-b)-(v-y)(b-y)}}+\eta$,

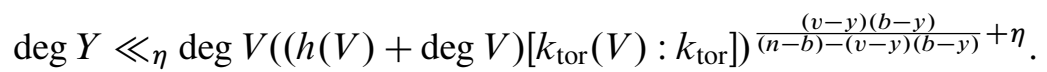

In addition the torsion points $\zeta$ belong to a finite set.

Proof of Theorem 7.1, part (1). Let $Y$ be a maximal $V$-torsion anomalous point with $B+\zeta$ minimal for $Y$.

We proceed to bound $\operatorname{deg} B$ and, in turn, the height of $Y$ and the degree of its field of definition. To this aim we use the Lehmer bound in Theorem 2.8 and the arithmetic Bézout theorem.

Let $v=\operatorname{dim} V$ and $b=\operatorname{dim} B$. By Lemma 2.2, $Y$ is a component of $V \cap B^{\prime}$ where $B^{\prime}$ is, like in the proof of Theorem 1.4, the zero component of the torsion variety defined by the first $v$ rows $h_{1}, \ldots, h_{v}$ of the matrix of $B$. Then codim $B^{\prime}=v$ and $\operatorname{deg} B^{\prime} \ll(\operatorname{deg} B)^{v /(n-b)}$.

We apply the arithmetic Bézout theorem to $V \cap B^{\prime}$ to obtain

$$
\hat{h}(Y) \ll \frac{(h(V)+\operatorname{deg} V)\left[k_{\mathrm{tor}}(V): k_{\mathrm{tor}}\right]}{\left[k_{\mathrm{tor}}(Y): k_{\mathrm{tor}}\right]}(\operatorname{deg} B)^{v /(n-b)} .
$$

Applying the Lehmer estimate in Theorem 2.8 to $Y$ in $B+\zeta$, instead, we have that for every positive real $\eta$,

$$
\hat{h}(Y) \gg_{\eta} \frac{(\operatorname{deg} B)^{1 / b-\eta}}{\left[k_{\mathrm{tor}}(Y): k_{\mathrm{tor}}\right]^{1 / b+\eta}} .
$$


From (23) and (24), and for $\eta$ small enough, we get the bound for $\operatorname{deg} B$ if $b>1$. If $b=1$ we use Bézout's theorem to bound the factor $\left[k_{\mathrm{tor}}(Y): k_{\mathrm{tor}}\right]^{\eta}$ as $\left((\operatorname{deg} B)(\operatorname{deg} V)\left[k_{\text {tor }}(V): k_{\text {tor }}\right]\right)^{\eta}$.

We then apply Bézout's theorem in $V \cap B^{\prime}$ to bound $\left[k_{\text {tor }}(Y): k_{\text {tor }}\right]$ and the arithmetic Bézout theorem in $V \cap B^{\prime}$ to prove the bound for $\hat{h}(Y)$. Finally, from Proposition 3.5 it follows that the points $\zeta$ belong to a finite set of cardinality effectively bounded.

Proof of Theorem 7.1, part (2). Let $Y=H+Y_{0}$ be a maximal $V$-torsion anomalous translate of positive dimension with minimal $B+\zeta$; assume also that $Y_{0} \in H^{\perp}$.

We use the Lehmer bound and the arithmetic Bézout theorem to bound deg $B$ and, in turn, the height and the degree of $H+Y_{0}$. In view of Remark 2.4, without loss of generality, we can assume that $\zeta=0$.

Let $b=\operatorname{dim} B, v=\operatorname{dim} V$ and $y=\operatorname{dim} Y=\operatorname{dim} H$. Clearly $v-y<n-b$ because $Y$ is torsion anomalous.

As before, by Lemma 2.2 we have that $Y$ is a component of $V \cap B^{\prime}$, where $B^{\prime}$ is an irreducible torsion variety with $\operatorname{codim} B^{\prime}=v-y$ and $\operatorname{deg} B^{\prime} \ll(\operatorname{deg} B)^{(v-y) /(n-b)}$. Arguing as usual on the conjugates of $H+Y_{0}$ over $k_{\text {tor }}(V)$, we see that the intersection $V \cap B^{\prime}$ has at least $\left[k_{\text {tor }}\left(H+Y_{0}\right): k_{\text {tor }}\right] /\left[k_{\text {tor }}(V): k_{\text {tor }}\right]$ components.

We apply the arithmetic Bézout theorem to the intersection $V \cap B^{\prime}$, obtaining

$$
h\left(H+Y_{0}\right) \ll(h(V)+\operatorname{deg} V)(\operatorname{deg} B)^{(v-y) /(n-b)} \frac{\left[k_{\mathrm{tor}}(V): k_{\mathrm{tor}}\right]}{\left[k_{\mathrm{tor}}\left(H+Y_{0}\right): k_{\mathrm{tor}}\right]} .
$$

By Zhang's inequality, Lemma 2.3 and (25), we deduce

$$
\mu\left(H+Y_{0}\right)=\mu\left(Y_{0}\right) \ll \frac{(h(V)+\operatorname{deg} V)\left[k_{\mathrm{tor}}(V): k_{\mathrm{tor}}\right](\operatorname{deg} B)^{(v-y) /(n-b)}}{\left[k_{\mathrm{tor}}\left(H+Y_{0}\right): k_{\mathrm{tor}}\right] \operatorname{deg} H} .
$$

For the lower bound for $\mu\left(Y_{0}\right)$, the proof follows the case of $\operatorname{dim} Y=0$. Let $H_{0}=H^{\perp} \cap B$. By minimality of $B$ we have that $H_{0}$ is a torsion variety of minimal dimension containing $Y_{0}$, thus

$$
\operatorname{dim} H_{0}=\operatorname{dim} H^{\perp}+\operatorname{dim} B-n=(n-y)+b-n=b-y .
$$

As in Theorem 1.4, part (3), one can easily see that

$$
\left[k_{\text {tor }}\left(Y_{0}\right): k_{\text {tor }}\right] \leq\left[k_{\text {tor }}\left(H+Y_{0}\right): k_{\text {tor }}\right] \cdot \#\left(H \cap H_{0}\right) .
$$

By the definition of $H_{0}$, we have $B=H+H_{0}$ and from [Masser and Wüstholz 1993, Lemma 1.2], we get

$$
\#\left(H \cap H_{0}\right) \operatorname{deg} B \leq \operatorname{deg} H \operatorname{deg} H_{0} .
$$


In addition, $H \cap H_{0} \subseteq H \cap H^{\perp}$, thus

$$
\#\left(H \cap H_{0}\right) \ll(\operatorname{deg} H)^{2} .
$$

Applying Theorem 2.8 to $Y_{0}$ in $H_{0}$ we get that, for every positive real $\eta$,

$$
\mu\left(Y_{0}\right)=\hat{h}\left(Y_{0}\right) \gg_{\eta} \frac{\left(\operatorname{deg} H_{0}\right)^{1 /(b-y)-\eta}}{\left[k_{\mathrm{tor}}\left(Y_{0}\right): k_{\mathrm{tor}}\right]^{1 /(b-y)+\eta}} .
$$

Combining (26) and (30), we have

$$
\frac{\left(\operatorname{deg} H_{0}\right)^{1 /(b-y)-\eta}}{\left[k_{\mathrm{tor}}\left(Y_{0}\right): k_{\mathrm{tor}}\right]^{1 /(b-y)+\eta}} \ll_{\eta} \frac{(h(V)+\operatorname{deg} V)\left[k_{\mathrm{tor}}(V): k_{\mathrm{tor}}\right](\operatorname{deg} B)^{(v-y) /(n-b)}}{\left[k_{\mathrm{tor}}\left(H+Y_{0}\right): k_{\mathrm{tor}}\right] \operatorname{deg} H},
$$

and hence, using (27)-(29) as in Theorem 1.4, part (3), we get the bound for deg $B$; notice that if $b-y>1$, the argument is in fact simpler, as we don't need to deal with the $\left[k_{\text {tor }}\left(Y_{0}\right): k_{\text {tor }}\right]^{\eta}$ term.

Having obtained a bound for $\operatorname{deg} B$, the degree of $H+Y_{0}$ can be bounded by applying Bézout's theorem to the intersection $V \cap B^{\prime}$ and using $\operatorname{deg} B^{\prime} \ll$ $\operatorname{deg} B^{(v-y) /(n-b)}$. The bound for $h\left(H+Y_{0}\right)$, instead, is derived from (25) and the bound for $\operatorname{deg} B$. Finally, from Proposition 3.5 we conclude that the points $\zeta$ belong to a finite set of cardinality effectively bounded.

Proof of Theorem 7.1, part (3). Assume that $Y \subseteq V \cap(B+\zeta)$ is not a translate.

If $Y$ is transverse in $B+\zeta$, the proof of Theorem 1.4, part (1) easily adapts to this case as well, yielding the desired bounds; let us then assume that $Y$ is not transverse. Without loss of generality, we can assume $\zeta=0$ (see Remark 2.4). Then $Y$ is transverse in a translate $H_{1}+Y_{0} \subsetneq B$, with $Y_{0} \in H_{1}^{\perp}$ and $H_{1}$ of minimal dimension.

We define $H_{0}=B \cap H_{1}^{\perp}$ so that $B=H_{1}+H_{0}$ and

$$
\operatorname{deg} B=\operatorname{deg}\left(H_{1}+H_{0}\right) \leq \frac{\operatorname{deg} H_{1} \operatorname{deg} H_{0}}{\#\left(H_{1} \cap H_{0}\right)} .
$$

We set $y=\operatorname{dim} Y, v=\operatorname{dim} V, b=\operatorname{dim} B, h_{1}=\operatorname{dim} H_{1}$ and $h_{0}=\operatorname{dim} H_{0}=b-h_{1}$.

Writing $Y=Y_{1}+Y_{0}$, we have that $Y_{1} \subseteq H_{1}$ is transverse in $H_{1}$ because $Y$ is transverse in $H_{1}+Y_{0}$, and $Y_{0} \subseteq H_{0}$ is transverse in $H_{0}$ because $B$ is minimal for $Y$.

By definition $Y_{1} \subseteq H_{1}$ and $Y_{0} \in H_{1}^{\perp}$. From Lemma 2.3 and the definition of essential minimum, we get

$$
\mu(Y)=\mu\left(Y_{1}\right)+\hat{h}\left(Y_{0}\right) .
$$

As usual, the upper bound for $\mu(Y)$ is obtained using the arithmetic Bézout theorem in $V \cap B^{\prime}$ for some abelian variety $B^{\prime}$ constructed by deleting $v-y$ 
suitable rows from $B$. All conjugates of $Y$ are components of same height in $V \cap B^{\prime}$. This gives

$$
\mu(Y) \ll(h(V)+\operatorname{deg} V)(\operatorname{deg} B)^{(v-y) /(n-b)} \frac{\left[k_{\text {tor }}(V): k_{\text {tor }}\right]}{\operatorname{deg} Y\left[k_{\text {tor }}\left(Y_{1}+Y_{0}\right): k_{\text {tor }}\right]} .
$$

Moreover,

$$
\left[k_{\text {tor }}\left(Y_{1}+Y_{0}\right): k_{\text {tor }}\right] \#\left(H_{1} \cap H_{0}\right) \geq\left[k_{\text {tor }}\left(Y_{0}\right): k_{\text {tor }}\right]
$$

because for every $\sigma \in \operatorname{Gal}\left(\bar{k}_{\text {tor }} / k_{\text {tor }}\right)$ which fixes $Y_{1}+Y_{0}$, the difference $\sigma\left(Y_{0}\right)-Y_{0}$ lies in $H_{1} \cap H_{0}$.

To obtain a lower bound for $\mu(Y)$ we either apply the Bogomolov bound to $Y_{1}$ in $H_{1}$ or the Lehmer estimate to $Y_{0}$ in $H_{0}$. These give

$$
\frac{\left(\operatorname{deg} H_{1}\right)^{1 /\left(h_{1}-y\right)-\eta}}{(\operatorname{deg} Y)^{1 /\left(h_{1}-y\right)+\eta}} \ll_{\eta} \mu\left(Y_{1}\right) \leq \mu(Y)
$$

and

$$
\frac{\left(\operatorname{deg} H_{0}\right)^{1 / h_{0}-\eta}}{\left[k_{\mathrm{tor}}\left(Y_{0}\right): k_{\mathrm{tor}}\right]^{1 / h_{0}+\eta}} \ll_{\eta} \hat{h}\left(Y_{0}\right) \leq \mu(Y) .
$$

We now relate the left-hand side to $\operatorname{deg} B$. Notice that either

$$
(\operatorname{deg} B)^{\left(h_{1}-y\right) /(b-y)}<\operatorname{deg} H_{1}
$$

or

$$
(\operatorname{deg} B)^{h_{0} /(b-y)} \leq \frac{\operatorname{deg} H_{0}}{\#\left(H_{1} \cap H_{0}\right)} .
$$

Indeed if (i) and (ii) were both false, then

$$
\operatorname{deg} B=(\operatorname{deg} B)^{\frac{h_{1}-y}{b-y}+\frac{h_{0}}{b-y}}>\frac{\operatorname{deg} H_{1} \operatorname{deg} H_{0}}{\#\left(H_{1} \cap H_{0}\right)},
$$

which contradicts (31).

Assume that (i) holds. Then (32), (34), (i) and the fact that $n-b>(v-y) \cdot(b-y)$ give the bound

$$
\operatorname{deg} B \ll_{\eta}\left((h(V)+\operatorname{deg} V)\left[k_{\mathrm{tor}}(V): k_{\mathrm{tor}}\right]\right)^{\frac{(b-y)(n-b)}{(n-b)-(v-y)(b-y)}+\eta},
$$

where, if $h_{1}-y=1$, the factor $(\operatorname{deg} Y)^{\eta}$ has been removed by applying Bézout's theorem to $Y$ in $V \cap B$ and changing $\eta$.

Assume that (ii) holds. Then (32), (35) (ii), the fact that $n-b>(v-y)(b-y)$ and (33) give the bound

$$
\operatorname{deg} B \ll_{\eta}\left((h(V)+\operatorname{deg} V)\left[k_{\mathrm{tor}}(V): k_{\mathrm{tor}}\right]\right)^{\frac{(b-y)(n-b)}{(n-b)-(v-y)(b-y)}+\eta},
$$


where, if $h_{0}=1$ the dependence on $\left[k_{\text {tor }}\left(Y_{0}\right): k_{\text {tor }}\right]$ can be removed using (33), bounding $\left[k_{\text {tor }}(Y): k_{\text {tor }}\right]$ as $\left[k_{\text {tor }}(V): k_{\text {tor }}\right] \operatorname{deg} V \operatorname{deg} B$ by the Bézout theorem applied to $Y$ in $V \cap B$ and observing that

$$
\#\left(H_{1} \cap H_{0}\right) \leq \#\left(H_{1} \cap H_{1}^{\perp}\right) \ll\left(\operatorname{deg} H_{1}\right)^{2} \ll(\operatorname{deg} Y)^{2 h_{1}} \leq(\operatorname{deg} V \operatorname{deg} B)^{2 h_{1}}
$$

because, since $Y_{1}$ is transverse in $H_{1}$, we have $H_{1}=Y_{1}+\cdots+Y_{1}\left(h_{1}\right.$ times), from which $\operatorname{deg} H_{1} \ll(\operatorname{deg} Y)^{h_{1}}$.

So we have bounded $\operatorname{deg} B$. We obtain the bounds for $\operatorname{deg} Y$ and $h(Y)$ applying respectively the Bézout Theorem and the arithmetic Bézout theorem to the intersection $Y \subseteq V \cap B^{\prime}$. Finally, Proposition 3.5 guarantees that the points $\zeta$ belong to a finite set of cardinality effectively bounded.

\section{Acknowledgements}

We thank Francesco Veneziano for an accurate revision of an earlier version of this paper. We kindly thank the referee for his useful comments and corrections. Especially, we thank him for pointing out the effectivity question of Proposition 3.5.

\section{References}

[Bertrand 1986] D. Bertrand, "Relations d'orthogonalité sur les groupes de Mordell-Weil", pp. 33-39 in Séminaire de théorie des nombres, Paris 1984-85, edited by C. Goldstein, Progr. Math. 63, Birkhäuser, Boston, 1986. MR 88h:11037 Zbl 0607.14014

[Bombieri and Zannier 1996] E. Bombieri and U. Zannier, "Heights of algebraic points on subvarieties of abelian varieties", Ann. Scuola Norm. Sup. Pisa Cl. Sci. (4) 23:4 (1996), 779-792. MR 98j:11043 Zbl 0897.11020

[Bombieri et al. 2007] E. Bombieri, D. Masser, and U. Zannier, "Anomalous subvarieties: structure theorems and applications", Int. Math. Res. Not. 2007:19 (2007), Art. ID \#rnm057. MR 2008k:11060 Zbl 1145.11049

[Carrizosa 2009] M. Carrizosa, "Petits points et multiplication complexe", Int. Math. Res. Not. 2009:16 (2009), 3016-3097. MR 2011c:11102 Zbl 1176.11025

[Checcoli et al. 2012] S. Checcoli, F. Veneziano, and E. Viada, "A sharp Bogomolov-type bound", New York J. Math. 18 (2012), 891-910. MR 2991428 Zbl 1276.11099

[Checcoli et al. 2014] S. Checcoli, F. Veneziano, and E. Viada, "On torsion anomalous intersections", Atti Accad. Naz. Lincei Rend. Lincei Mat. Appl. 25:1 (2014), 1-36. MR 3180478 Zbl 06293297

[David and Philippon 2002] S. David and P. Philippon, "Minorations des hauteurs normalisées des sous-variétés de variétés abeliennes, II”, Comment. Math. Helv. 77:4 (2002), 639-700. MR 2004a: 11055 Zbl 1030.11026

[Galateau 2010] A. Galateau, "Une minoration du minimum essentiel sur les variétés abéliennes", Comment. Math. Helv. 85:4 (2010), 775-812. MR 2011i:11110 Zbl 1250.11071

[Habegger 2008] P. Habegger, "Intersecting subvarieties of $\mathbf{G}_{m}^{n}$ with algebraic subgroups", Math. Ann. 342:2 (2008), 449-466. MR 2009f:14044 Zbl 1168.14019

[Masser and Wüstholz 1993] D. Masser and G. Wüstholz, "Periods and minimal abelian subvarieties", Ann. of Math. (2) 137:2 (1993), 407-458. MR 94g:11040 Zbl 0796.11023 
[Maurin 2008] G. Maurin, "Courbes algébriques et équations multiplicatives", Math. Ann. 341:4 (2008), 789-824. MR 2009g:14026 Zbl 1154.14017

[Philippon 1991] P. Philippon, "Sur des hauteurs alternatives, I", Math. Ann. 289:2 (1991), 255-283. MR 92m:11061 Zbl 0726.14017

[Philippon 1995] P. Philippon, "Sur des hauteurs alternatives, III", J. Math. Pures Appl. (9) 74:4 (1995), 345-365. MR 97a:11098 Zbl 0878.11025

[Philippon 2012] P. Philippon, "Sur une question d'orthogonalité dans les puissances de courbes elliptiques", preprint, Institut de Mathématiques de Jussieu, 2012, http://hal.archives-ouvertes.fr/ hal-00801376.

[Rémond 2000] G. Rémond, "Décompte dans une conjecture de Lang”, Invent. Math. 142:3 (2000), 513-545. MR 2002f:14058 Zbl 0972.11054

[Shafarevich 1972] I. R. Shafarevich, Basic algebraic geometry, 1: Varieties in projective space, Springer, Heidelberg, 1972. MR 3100243 Zbl 0284.14001

[Viada 2008] E. Viada, "The intersection of a curve with a union of translated codimensiontwo subgroups in a power of an elliptic curve", Algebra Number Theory 2:3 (2008), 249-298. MR 2009f:11079 Zbl 1168.11024

[Zhang 1998] S.-W. Zhang, "Equidistribution of small points on abelian varieties", Ann. of Math. (2) 147:1 (1998), 159-165. MR 99e:14032 Zbl 0991.11034

Received July 10, 2013. Revised May 6, 2014.

\section{SARA CHECCOLI}

INSTITUT FOURIER

UNIVERSITÉ JOSEPH FOURIER, GRENOBLE

100 RUE DES MATHS

38402 ST MARTIN D'HÈRES

FRANCE

sara.checcoli@ujf-grenoble.fr

EVELINA VIADA

Mathematisches InstituT

GEORG-AUGUST-UNIVERSITÄT

BUNSENSTRASSE 3-5

D-D-37073 GÖTTINGEN

GERMANY

evelina.viada@unibas.ch 


\title{
PACIFIC JOURNAL OF MATHEMATICS
}

\author{
msp.org/pjm
}

Founded in 1951 by E. F. Beckenbach (1906-1982) and F. Wolf (1904-1989)

\section{EDITORS}

Don Blasius (Managing Editor)

Department of Mathematics

University of California

Los Angeles, CA 90095-1555

blasius@math.ucla.edu

\author{
Paul Balmer \\ Department of Mathematics \\ University of California \\ Los Angeles, CA 90095-1555 \\ balmer@math.ucla.edu \\ Robert Finn \\ Department of Mathematics \\ Stanford University \\ Stanford, CA 94305-2125 \\ finn@math.stanford.edu \\ Sorin Popa \\ Department of Mathematics \\ University of California \\ Los Angeles, CA 90095-1555 \\ popa@math.ucla.edu
}

\author{
Vyjayanthi Chari \\ Department of Mathematics \\ University of California \\ Riverside, CA 92521-0135 \\ chari@math.ucr.edu \\ Kefeng Liu \\ Department of Mathematics \\ University of California \\ Los Angeles, CA 90095-1555 \\ liu@math.ucla.edu \\ Jie Qing \\ Department of Mathematics \\ University of California \\ Santa Cruz, CA 95064 \\ qing@ cats.ucsc.edu
}

\section{PRODUCTION}

Silvio Levy, Scientific Editor, production@msp.org

\section{SUPPORTING INSTITUTIONS}

ACADEMIA SINICA, TAIPEI

CALIFORNIA INST. OF TECHNOLOGY

INST. DE MATEMÁTICA PURA E APLICADA

KEIO UNIVERSITY

MATH. SCIENCES RESEARCH INSTITUTE

NEW MEXICO STATE UNIV.

OREGON STATE UNIV.

\author{
STANFORD UNIVERSITY \\ UNIV. OF BRITISH COLUMBIA \\ UNIV. OF CALIFORNIA, BERKELEY \\ UNIV. OF CALIFORNIA, DAVIS \\ UNIV. OF CALIFORNIA, LOS ANGELES \\ UNIV. OF CALIFORNIA, RIVERSIDE \\ UNIV. OF CALIFORNIA, SAN DIEGO \\ UNIV. OF CALIF., SANTA BARBARA
}

\author{
Daryl Cooper \\ Department of Mathematics \\ University of California \\ Santa Barbara, CA 93106-3080 \\ cooper@math.ucsb.edu \\ Jiang-Hua Lu \\ Department of Mathematics \\ The University of Hong Kong \\ Pokfulam Rd., Hong Kong \\ jhlu@maths.hku.hk \\ Paul Yang \\ Department of Mathematics \\ Princeton University \\ Princeton NJ 08544-1000 \\ yang@math.princeton.edu
}

These supporting institutions contribute to the cost of publication of this Journal, but they are not owners or publishers and have no responsibility for its contents or policies.

See inside back cover or msp.org/pjm for submission instructions.

The subscription price for 2014 is US $\$ 410 /$ year for the electronic version, and \$535/year for print and electronic.

Subscriptions, requests for back issues and changes of subscribers address should be sent to Pacific Journal of Mathematics, P.O. Box 4163, Berkeley, CA 94704-0163, U.S.A. The Pacific Journal of Mathematics is indexed by Mathematical Reviews, Zentralblatt MATH, PASCAL CNRS Index, Referativnyi Zhurnal, Current Mathematical Publications and Web of Knowledge (Science Citation Index).

The Pacific Journal of Mathematics (ISSN 0030-8730) at the University of California, c/o Department of Mathematics, 798 Evans Hall \#3840, Berkeley, CA 94720-3840, is published twelve times a year. Periodical rate postage paid at Berkeley, CA 94704, and additional mailing offices. POSTMASTER: send address changes to Pacific Journal of Mathematics, P.O. Box 4163, Berkeley, CA 94704-0163.

PJM peer review and production are managed by EditFLOW ${ }^{\circledR}$ from Mathematical Sciences Publishers.

\section{PUBLISHED BY}

\section{mathematical sciences publishers \\ nonprofit scientific publishing}

http://msp.org/

(C) 2014 Mathematical Sciences Publishers 


\section{PACIFIC JOURNAL OF MATHEMATICS}

Volume $271 \quad$ No. $2 \quad$ October 2014

Monoids of modules and arithmetic of direct-sum decompositions

NiChOlas R. BAETH and AlFRED GEROLDINGER

On the torsion anomalous conjecture in $\mathrm{CM}$ abelian varieties

SARA CHECCOLI and EVELINA VIADA

Eigenvalue estimate and compactness for closed $f$-minimal surfaces

347

Xu Cheng, Tito Mejia and Detang Zhou

Lefschetz numbers of symplectic involutions on arithmetic groups

369

STEFFEN KIONKE

Categorification of a parabolic Hecke module via sheaves on moment 415 graphs

MARTINA LANINI

Unitary representations of $\operatorname{GL}(n, K)$ distinguished by a Galois

445 involution for a $p$-adic field $K$

NADIR MATRINGE

On $f$-biharmonic maps and $f$-biharmonic submanifolds

461

YE-LIN OU

Unitary principal series of split orthogonal groups

Alessandra Pantano, AnNegret Paul and Susana SALAMANCA RIBA 